\title{
Purification and kinetic analysis of a baculovirus ecdysteroid UDP-glucosyltransferase
}

\author{
Owain P. EVANS and David R. O'REILLY' \\ Department of Biology, Imperial College of Science, Technology, and Medicine, Prince Consort Road, London SW7 2BB, U.K.
}

The baculovirus ecdysteroid UDP-glucosyltransferase (EGT) disrupts the hormonal balance of the insect host by catalysing the conjugation of ecdysteroids, the moulting hormones, with the sugar moiety from UDP-glucose or UDP-galactose. In this study, Autographa californica nucleopolyhedrovirus EGT has been overproduced and purified, and its kinetic properties determined. The enzyme was purified 1100 -fold to nearhomogeneity using only two major steps, ion-exchange and gel-filtration chromatography. EGT activity was eluted from the gel-filtration column as a single peak corresponding to a $260 \pm 50 \mathrm{kDa}$ protein, suggesting that the enzyme is an oligomer of three to five subunits, as the subunit molecular mass is approximately $56 \mathrm{kDa}$. Kinetic analysis showed that EGT has broadly similar specificities for UDP-galactose and UDP-glucose ( $k_{\text {cat }} / K_{\mathrm{m}}=1790.8$ and 902.1 respectively) when ecdysone is used as the other substrate. On the other hand, it shows marked differences in specificity for the various ecdysteroids tested. Ecdysone seems to be the optimal substrate $\left(k_{\text {cat }} / K_{\mathrm{m}}=7101.1\right)$, whereas 3-dehydroecdysone, an ecdysone precursor in Lepidoptera, is seven times less favourable $\left(k_{\text {cat }} / K_{\mathrm{m}}=1085.7\right)$. Notably, 20-hydroxyecdysone, the active form of the hormone, is conjugated very poorly $\left(k_{\text {cat }} / K_{\mathrm{m}}=31.6\right)$. Analysis of the data revealed that the enzyme mechanism involves the formation of an ecdysteroid-UDP-sugar-enzyme ternary complex. This work represents the most detailed biochemical characterization of an EGT to date.

\section{INTRODUCTION}

Baculoviruses are a large and diverse family of viruses which infect mainly insect species, most commonly those of the order Lepidoptera. They are large DNA viruses, having a doublestranded covalently closed circular genome of between 80 and $200 \mathrm{kbp}$. In general, baculoviruses infect the larval stages of their insect host. A remarkable feature of the virus-host interaction is that the virus is able to arrest the development of an infected insect, preventing it from moulting or pupating [1]. By prolonging the time in which the host is in an actively feeding larval stage, the virus ensures that the infected host is larger than it would otherwise be and therefore yields more progeny virus [2].

We have shown that developmental arrest is brought about by the action of a virus-encoded ecdysteroid UDP-glucosyltransferase (EGT) [1]. Moulting and pupation in Lepidoptera are under the control of a family of steroid hormones known as ecdysteroids (reviewed in [3]). During viral infection, EGT is secreted from infected cells into the insect's haemolymph where it catalyses the conjugation of circulating ecdysteroids with glucose or galactose, donated by the respective UDP-sugars [4]. This blocks the induction of ecdysis.

A large number of baculovirus egt genes have now been identified (reviewed in [5]). Of these, the Autographa californica nucleopolyhedrovirus (AcMNPV) egt is the best characterized. The ecdysteroid and UDP-sugar specificities of the enzyme have been investigated, and we have shown that the product generated after conjugation of ecdysone with glucose is ecdysone $22-O-\beta$-Dglucopyranoside [6]. However, no further information is available on the kinetic properties or mode of action of this enzyme.

To date, egt genes have only been identified in baculovirus genomes, but there is clear evidence that insects possess a similar gene. The ecdysteroid conjugate 26-hydroxyecdysone-22-glucoside has been isolated from Manduca sexta (tobacco horn- worm) embryos [7,8]. Since conjugation in this instance is also at the C-22 position of the ecdysteroid, it is likely that the insect enzyme is very similar to that encoded by the virus. It seems possible that baculoviruses acquired the egt gene from the insect host during the course of their evolution. egt genes do not appear to be restricted to insects; for example, ecdysteroid glucosides are known to be products of ecdysteroid metabolism in the nematode Parascaris equorum (reviewed in [9]).

The baculovirus EGTs, and presumably other invertebrate EGTs, belong to a much larger family of transferases that conjugate small lipophilic compounds with various sugars [1]. In particular, EGT exhibits significant amino acid similarity to the mammalian UDP-glucuronosyltransferases (UDPGTs) [1]. These enzymes play a central role in the detoxication and elimination of a wide range of endogenous and exogenous compounds [10]. Conjugation with glucuronic acid increases the polarity of the aglycone, facilitating its removal by excretion. The principal endogenous compounds metabolized in this way are the bile acids, bilirubin and certain steroid hormones. The importance of these conjugation reactions is underlined by the fact that a number of serious pathologies are associated with lesions in UDPGT genes. In particular, the severe disorder Crigler-Najjar syndrome is known to be caused by mutation of the gene encoding bilirubin UDP-glucuronosyltransferase [11]. In this disease, bilirubin is not removed and accumulates to toxic levels in the blood. Patients suffering from Crigler-Najjar syndrome generally succumb in early childhood.

The UDPGTs are membrane-bound enzymes, with the body of the protein protruding into the lumen of the endoplasmic reticulum (ER). Because of this, they have proved to be very difficult to purify; the use of detergents needed to liberate the tightly bound enzymes from the ER membranes generally leads to inactivation of the enzyme or to very low yields of functional enzyme. Furthermore, owing to the intimate relationship of

Abbreviations used: AcMNPV, Autographa californica nucleopolyhedrovirus; 3DE, 3-dehydroecdysone; 20E, 20-hydroxyecdysone; EGT, ecdysteroid UDP-glucosyltransferase; ER, endoplasmic reticulum; LdMNPV, Lymantria dispar nucleopolyhedrovirus; UDPGT, UDP-glucuronosyltransferase.

1 To whom correspondence should be addressed. 
these enzymes with phospholipids [12], the extraction of the UDPGTs from the ER membranes frequently causes erratic changes in their stability and substrate specificity [13]. The UDPGTs are retained in the ER membrane by an 'anchor' sequence located at the extreme C-terminus [10]. EGT is secreted because it lacks this sequence. It can thus be readily purified, and we suggest that it represents a useful model system for the investigation of the biochemistry of this group of enzymes. In this study, we have overexpressed and purified AcMNPV EGT and assessed several of its biochemical characteristics. Its optimal $\mathrm{pH}$ range has been determined, and quantitative kinetic measurements have been made with a variety of UDP-sugar and ecdysteroid substrates. Our data show that EGT-catalysed conjugation proceeds via the formation of a ternary enzymeecdysteroid-UDP-sugar intermediate. This work thus provides a significant insight into the catalytic properties of this important family of enzymes.

\section{EXPERIMENTAL}

\section{Chemicals (including radiochemicals) and reagents}

These were supplied by Sigma (U.K.), unless noted otherwise. 3Dehydroecdysone (3DE) was kindly supplied by Professor H. H. Rees (University of Liverpool, U.K.).

\section{Cells and viruses}

Cells were maintained as monolayer cultures in Nunc plastic tissue-culture flasks (Gibco-Life Technologies Ltd., Paisley, Scotland, U.K.). Spodoptera frugiperda IPLB-SF21 cells [14] were maintained in TC100 medium (Gibco-Life Technologies Ltd.) supplemented with $0.26 \%$ tryptose broth and $10 \%$ foetalcalf serum (MBM Ltd.) or in SF900 II serum-free medium (Gibco-Life Technologies Ltd.). The EGT-overproducing virus vSynEVEGT contains two copies of the egt gene, one at its normal site and a second at the polyhedrin locus under the control of a modified polyhedrin promoter (O. P. Evans and D. R. O'Reilly, unpublished work). This virus was propagated and titred on SF21 cells as described [15].

\section{Assay of EGT activity}

A novel method was used for the assay of EGT activity. Unless noted otherwise, the standard assay (total reaction volume as stated for the respective assays) included $0.05 \mu \mathrm{Ci}$ of $\left[{ }^{3} \mathrm{H}\right] \mathrm{UDP}-$ glucose (15.3 Ci/mmol), $20 \mu \mathrm{M}$ ecdysone, $10 \mathrm{mM} \mathrm{MgCl}_{2}, 10 \mathrm{mM}$ Tris/maleate, $\mathrm{pH} 7.4$, and an appropriate amount of enzyme (generally $0.1-0.6 \mu \mathrm{g}$ of purified EGT). Incubation was at $37^{\circ} \mathrm{C}$ for $30 \mathrm{~min}$, except where stated otherwise. The reaction was stopped and the conjugate extracted by addition of an equal volume of water-saturated butan-1-ol, followed by centrifugation at $15000 \mathrm{~g}$ for $7 \mathrm{~min}$ at $4{ }^{\circ} \mathrm{C}$. Around $50-63 \%$ of the conjugate (depending on the nature of the sugar and hormone molecules involved) partitions into the organic phase, whereas unincorporated UDP-sugar remains in the aqueous phase. The amount of radioactivity in the organic phase was determined. Calibration experiments were carried out to relate the amount of radioactivity present in the organic phase to the total amount of product formed. The standard deviation in the amount of product formed in independent assays with reasonable yield (e.g. more than 2 pmol of conjugate) was always less than $2 \%$ of the mean. Separate calibration experiments were carried out for each ecdysteroid-UDP-sugar combination to control for variation in partitioning of different conjugates (results not shown).

\section{Purification of EGT}

\section{Sample preparation}

SF21 cells $\left(3 \times 10^{8}\right)$ grown in SF900 II medium were infected with vSynEVEGT at a multiplicity of infection of 10 plaque-forming units per cell and incubated at $27^{\circ} \mathrm{C}$. The extracellular fluid (approx. $150 \mathrm{ml}$ ) was harvested 3 days later, centrifuged at $1000 \mathrm{~g}$ for $5 \mathrm{~min}$ and adjusted to $50 \mathrm{ng} / \mathrm{ml}$ leupeptin, $1 \mu \mathrm{g} / \mathrm{ml}$ pepstatin A, $100 \mu \mathrm{g} / \mathrm{ml} \mathrm{PMSF}$ and $0.4 \mathrm{mg} / \mathrm{ml} \mathrm{NaN}_{3}$. This solution was dialysed at $4{ }^{\circ} \mathrm{C}$ against 20 vol. of $10 \mathrm{mM}$ potassium acetate, pH 5.5 (including $0.04 \% \mathrm{NaN}_{3}$ ) with three changes of buffer. After clarification by centrifugation at $1000 \mathrm{~g}$ for $10 \mathrm{~min}$, the dialysate was made up to $300 \mathrm{ml}$ with $10 \mathrm{mM}$ potassium acetate, $\mathrm{pH} 5.5$.

\section{Ion-exchange chromatography}

The sample was applied at a flow rate of $8 \mathrm{ml} / \mathrm{h}$ to a CM52 CMcellulose column (Whatman) equilibrated in $25 \mathrm{mM}$ potassium acetate, $\mathrm{pH}$ 5.5. After the column had been washed with 5 column volumes of $25 \mathrm{mM}$ potassium acetate, $\mathrm{pH} 5.5$, bound material was eluted with a linear $0-500 \mathrm{mM} \mathrm{KCl}$ gradient made up in $10 \mathrm{mM}$ potassium acetate, $\mathrm{pH}$ 5.5. Fractions of volume $8 \mathrm{ml}$ were collected, and a $60 \mu \mathrm{l}$ aliquot of each was assayed for EGT activity in a volume of $600 \mu$ l. Protein concentration (using the BCA Protein Assay Kit; Pierce) and conductivity of each aliquot were also measured.

\section{Gel-filtration chromatography}

The five ion-exchange fractions with peak EGT activity were pooled and concentrated to $4 \mathrm{ml}$ using a YM30 ultrafiltration membrane mounted in a stirred pressure cell (Amicon). Then $1 \mathrm{ml}$ of the concentrate was adjusted to $5 \%(\mathrm{v} / \mathrm{v})$ glycerol and applied at $20 \mathrm{ml} / \mathrm{h}$ to a calibrated Sephacryl S-300 HR column (Pharmacia) previously equilibrated in $100 \mathrm{mM}$ potassium acetate, $\mathrm{pH}$ 5.5. Protein levels in the eluate were monitored by in-line recording of the $A_{280}$. Fractions of volume $3.3 \mathrm{ml}$ were collected and $15 \mu \mathrm{l}$ aliquots from each assayed for EGT activity in a volume of $300 \mu \mathrm{l}$. All fractions were stored at $4{ }^{\circ} \mathrm{C}$.

\section{Kinetic measurements}

Assays were performed using mixtures containing all possible combinations of five fixed concentrations of both a UDP-sugar [either $\left[{ }^{3} \mathrm{H}\right] \mathrm{UDP}$-glucose or $\left[{ }^{3} \mathrm{H}\right] \mathrm{UDP}$-galactose $\left.(10.6 \mathrm{Ci} / \mathrm{mmol})\right]$ and an ecdysteroid [ecdysone, 20-hydroxyecdysone (20E) or $3 \mathrm{DE}]$. The inverse was used to quantify the kinetic values for the UDP-sugars. The reaction (total reaction volume of $50 \mu \mathrm{l}$ ) contained $140 \mathrm{ng}$ of pure EGT, and was incubated at $37^{\circ} \mathrm{C}$ for 15 min [EGT was previously shown to generate product at a linear rate for at least $40 \mathrm{~min}$ (results not shown)] and the conjugate extracted and quantified as before. Rates were calculated using each ecdysteroid/UDP-sugar combination. Values for the kinetic constants $K_{\mathrm{m}}$ and $V_{\max }$ were obtained using Leonora, a program designed for the analysis of enzyme kinetic data [16].

\section{Measurement of pH optimum}

Aliquots of the EGT preparation were assayed in several different $100 \mathrm{mM}$ buffers with an overall range of $\mathrm{pH} 4-10.5$. The total reaction volume was $100 \mu \mathrm{l}$. The following buffers were used (for $\mathrm{pH}$ details, see legend to Figure 2); sodium acetate, Pipes, sodium phosphate, Tris/maleate, Tris $/ \mathrm{HCl}$ and glycine $/ \mathrm{NaOH}$. 


\section{SDS/PAGE}

SDS/PAGE was carried out using the discontinuous buffer system devised by Laemmli [17]. Protein samples were run on $10 \%$ polyacrylamide gels and visualized by staining with silver nitrate as described [18].

\section{RESULTS}

\section{Purification}

The recombinant AcMNPV baculovirus vSynEVEGT (O. P. Evans and D. R. O'Reilly, unpublished work) was used to overproduce EGT in cell culture. The enzyme was purified from the cell-culture medium by sequential ion-exchange and gelfiltration chromatography, as described (see the Experimental section). EGT was eluted from the ion-exchange column as a single peak at approx. $140 \mathrm{mM} \mathrm{KCl}$. Gel filtration of the concentrated peak fractions yielded a single distinct peak corresponding to a molecular mass of approx. $260 \pm 50 \mathrm{kDa}$. The progress of purification was monitored by SDS/PAGE analysis of samples from different stages of the procedure (Figure 1). EGT activity is seen to correspond to a diffuse band of approx. $56 \mathrm{kDa}$. On the basis of silver staining of the gels, we estimate that the final EGT preparation is at least $90 \%$ pure. Calculation of EGT specific activity at each stage of the protocol revealed that an overall purification of 1100 -fold was achieved, with a yield of $0.8 \%$ (Table 1 ).

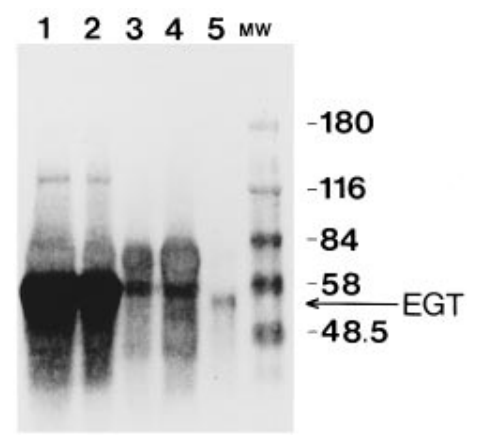

Figure 1 Silver-stained SDS/10\% polyacrylamide gel containing samples taken from various stages of the purification process

Equivalent volumes of the following samples were loaded in each lane: 1, cell-culture medium; 2, cell-culture medium dialysate; 3 , ion-exchange eluate; 4 , before gel filtration (concentrate); 5 , gel-filtration peak fraction. The sizes of the molecular-mass markers (MW) are shown in $\mathrm{kDa}$ down the right hand side of the gel. EGT (indicated by an arrow) is visible on the gel as a fuzzy band corresponding to a protein of approx. $56 \mathrm{kDa}$.

Table 1 Summary of EGT purification

One unit of activity corresponds to $1 \mathrm{pmol}$ of ecdysone-glucoside formed/min.

\begin{tabular}{lrlrlr}
\hline & $\begin{array}{l}\text { Total } \\
\text { protein } \\
\text { Sample }\end{array}$ & $\begin{array}{l}\text { Total } \\
\text { activity } \\
\text { (units) }\end{array}$ & $\begin{array}{l}\text { Specific } \\
\text { activity } \\
\text { (units/mg) }\end{array}$ & $\begin{array}{l}\text { Yield } \\
(\%)\end{array}$ & $\begin{array}{l}\text { Purification } \\
\text { factor }\end{array}$ \\
\hline Cell culture medium dialysate & 5091.68 & 3692.75 & 0.73 & 100 & 1 \\
lon-exchange eluate & 32.73 & 156.72 & 4.79 & 4.24 & 6.60 \\
Before gel filtration (concentrate) & 5.91 & 112.32 & 19.00 & 3.04 & 26.20 \\
Gel-filtration peak fraction & 0.04 & 29.45 & 785.13 & 0.80 & 1082.56 \\
& & & & & \\
\hline
\end{tabular}

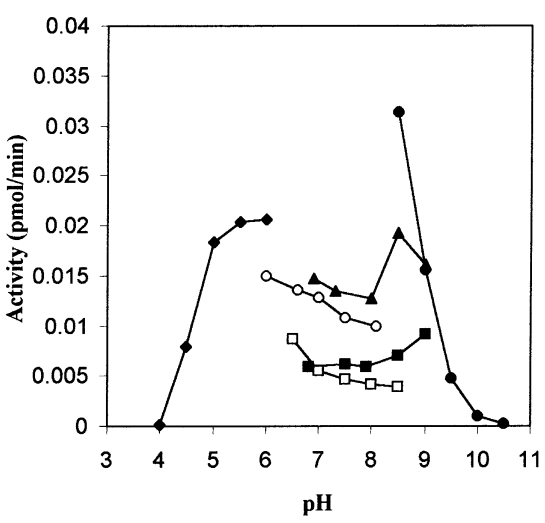

Figure 2 Estimation of the $\mathrm{pH}$ optimum of EGT

EGT activity was assayed in the following buffers: $100 \mathrm{mM}$ sodium acetate $(\bullet ; \mathrm{pH} 4.0,4.5$ 5.0, 5.5, 6.0), $100 \mathrm{mM}$ Pipes ( $\mathrm{O}$; pH 6.0, 6.5, 7.0, 7.5, 8.0), $100 \mathrm{mM}$ sodium phosphate $(\square ; \mathrm{pH} 6.5,7.0,7.5,8.0,8.5), 100 \mathrm{mM}$ Tris/maleate $(\square ; \mathrm{pH} \mathrm{6.8,7.5,7.9,8.5,9.0)}$, $100 \mathrm{mM}$ Tris/HCl $(\boldsymbol{\Delta} ; \mathrm{pH} 6.9,7.3,8.0,8.5,9.0)$ and $100 \mathrm{mM}$ glycine/ $\mathrm{NaOH}(\mathbf{O} ; \mathrm{pH} 8.5$, $9.0,9.5,10.0,10.5)$. EGT activity is expressed as pmol of ecdysone glucoside formed/min.

\section{Measurement of pH optimum}

Aliquots of the pure EGT preparation were assayed in a range of different buffers to estimate the $\mathrm{pH}$ optimum of the enzyme. It is evident that EGT is active over a very wide range; significant EGT activity was detected over the $\mathrm{pH}$ range 4-10.5 (Figure 2). Similarly, the enzyme seems to have a broad $\mathrm{pH}$ optimum, apparently in the range $\mathrm{pH}$ 6-8.5. Lepidopteran haemolymph (generally pH 6.4-6.8 [19]), the site of action of EGT, and insect cell culture medium $(\mathrm{pH}$ 6.2) both have $\mathrm{pH}$ values within this range.

\section{Kinetic data}

\section{Ecdysteroids}

We have previously shown that EGT can catalyse the conjugation of the ecdysteroids ecdysone, 20E, 26-hydroxyecdysone and makisterone A $[1,6]$. We have now shown that the ecdysteroid $3 \mathrm{DE}$ can be added to this list. One of the aims of this study was to determine the specificity of EGT for $3 \mathrm{DE}$, ecdysone and $20 \mathrm{E}$, the three molecules that are at the centre of lepidopteran ecdysteroid metabolism. The initial rates of the glucose conjugation of each of these ecdysteroids were measured; a sample double-reciprocal plot is shown in Figure 3(a). The kinetic constants, $K_{\mathrm{m}}$ and $V_{\max }$, and the specificity constant $k_{\text {cat }} / K_{\mathrm{m}}$ were quantified from these rates. The values of the specificity constants for these ecdysteroids show that EGT conjugates ecdysone approximately seven times more efficiently than 3DE, and utilizes the latter over 34 times more readily than 20E (Table 2). Since the $V_{\max }$ values for ecdysone and 3DE are not significantly different, the higher value of the specificity constant for the former is entirely attributable to the fact that EGT has an almost 7-fold higher affinity (as indicated by the value of $K_{\mathrm{m}}$ ) for ecdysone. In contrast, EGT has a very low specificity for $20 \mathrm{E}$ when compared with both ecdysone and 3DE. The most significant factor contributing to this low efficiency of conjugation is the markedly reduced $V_{\max }$ for 20E. The affinity of EGT for this substrate also appears to be quite low. However, because some assays with this substrate yielded low amounts of conjugate, the errors associated with these assays were relatively large, resulting in significant errors in the values of the kinetic constants. For this 
(a)

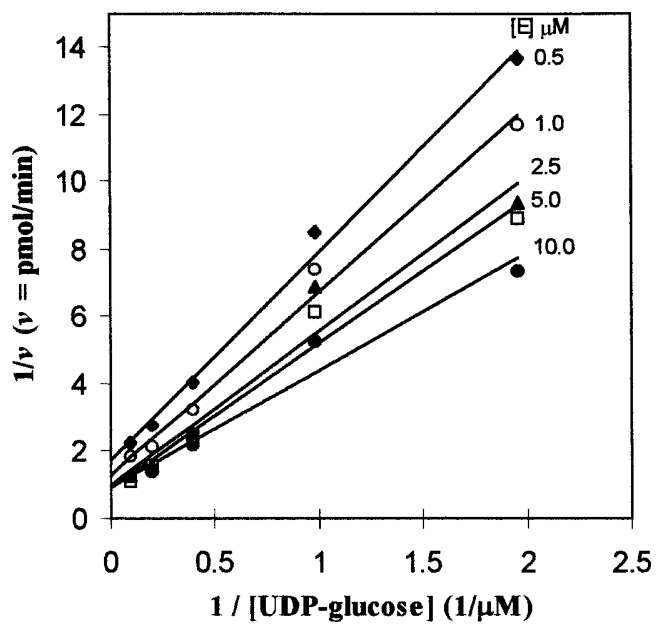

(b)

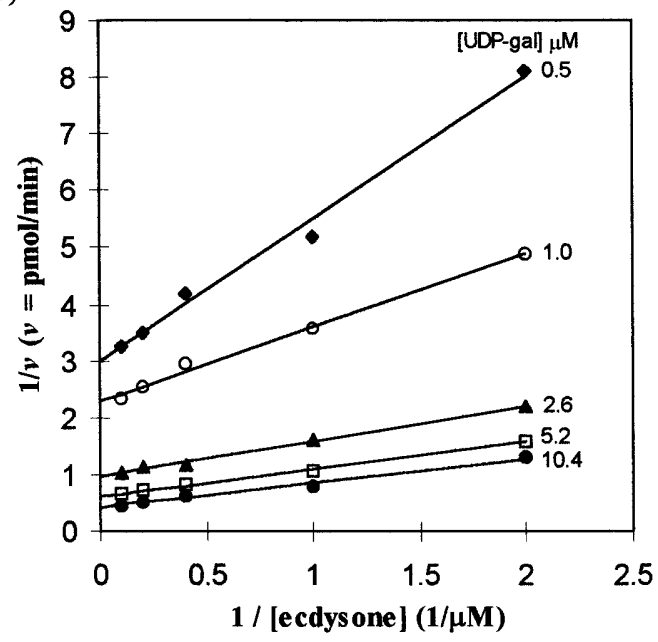

Figure 3 Sample Lineweaver-Burk plots of initial reaction rates as a function of either (a) ecdysone (E) or (b) UDP-galactose concentration

Reaction conditions are described in the Experimental section.

Table 2 Results of quantitative kinetic measurements made on EGTcatalysed reactions when a variety of ecdysteroid and UDP-sugar substrates were being used

The values of the kinetic constants $V_{\max }$ and $K_{m}$ were calculated using Leonora, a program for the analysis of enzyme kinetic data [16], and are means \pm S.E.M. Values for $k_{\text {cat }}$ and the specificity constant, $k_{\text {cat }} / K_{\mathrm{m}}$, are also shown for each substrate. The values for $k_{\text {cat }}$ were calculated assuming that the molecular mass of EGT is $260 \mathrm{kDa}$.

\begin{tabular}{lllcc}
\hline & $\begin{array}{l}V_{\max } \\
(\mathrm{pmol} / \mathrm{min})\end{array}$ & $\begin{array}{l}K_{\mathrm{m}} \\
(\mu \mathrm{M})\end{array}$ & $\begin{array}{l}10^{3} \times k_{\text {cat }} \\
\left(\mathrm{min}^{-1}\right)\end{array}$ & $\begin{array}{l}k_{\text {cat }} / K_{\mathrm{m}} \\
\left(\text { litre } \cdot \mathrm{min}^{-1} \cdot \mathrm{mol}^{-1}\right)\end{array}$ \\
\hline $\begin{array}{llll}\text { Ecdysteroids } \\
\quad 3 D E\end{array}$ & $1.68 \pm 0.48$ & $5.36 \pm 2.30$ & 5.82 & 1085.74 \\
$\quad$ Ecdysone & $1.66 \pm 0.13$ & $0.81 \pm 0.16$ & 5.75 & 7101.13 \\
20E & $0.12 \pm 0.11$ & $12.73 \pm 15.60$ & 0.40 & 31.60 \\
UDP-sugars & & & & \\
$\quad$ UDP-Glc & $1.66 \pm 0.13$ & $6.37 \pm 0.52$ & 5.75 & 902.13 \\
UDP-Gal & $4.15 \pm 0.28$ & $8.03 \pm 0.90$ & 14.37 & 1790.76 \\
& & & & \\
\hline
\end{tabular}

reason, it is difficult to evaluate whether the $K_{\mathrm{m}}$ for $20 \mathrm{E}$ is significantly higher than that for either ecdysone or $3 \mathrm{DE}$.

\section{UDP-sugars}

EGT can catalyse reactions involving more than one type of UDP-sugar; UDP-glucose and UDP-galactose have both been shown to be sugar donors in vitro [4]. The kinetic constants for the conjugation of ecdysone with the sugar moieties from either of these UDP-sugars were quantified. An example of a doublereciprocal plot, in this case showing the effect of UDP-galactose concentration on the rate of conjugation, is shown in Figure 3(b). The values for the specificity constants imply that EGT uses UDP-galactose approximately twice as efficiently as UDPglucose. This is because the enzyme exhibits a larger $V_{\max }$ with UDP-galactose, partly offset by a slightly greater affinity for UDP-glucose (Table 2).

\section{DISCUSSION}

In this study, the enzyme EGT from the baculovirus AcMNPV was purified approx. 1100-fold to near-homogeneity (Table 1). Since EGT is a secreted protein, and can be expressed in cells grown in serum-free medium, a pure enzyme preparation can be obtained with comparative ease, because of the relative purity of the starting material. In this study, we have demonstrated that EGT can be purified to at least $90 \%$ homogeneity using only two major steps, ion-exchange and gel-filtration chromatography.

SDS/PAGE analysis of the peak gel-filtration fraction (Figure 1) demonstrated that EGT migrates as a protein of approx. $56 \mathrm{kDa}$. This is in agreement with previous data from pulselabelling of infected cells showing that EGT has a molecular mass of approx. $60 \mathrm{kDa}$ in denaturing conditions [20]. In contrast, however, in this study EGT activity was eluted from gel-filtration columns as a peak corresponding to an approximate molecular mass of $260 \pm 50 \mathrm{kDa}$. This suggests that, in its native state, EGT is an oligomeric enzyme, consisting of between three and five subunits. This matter is to be investigated further. Nevertheless, it is of interest to note that the mammalian UDPGTs have been shown to consist of between one and six subunits (each monomer being typically $50-60 \mathrm{kDa}$ ), depending on the enzyme (for examples see [21-23]).

Previous studies indicated that EGT could conjugate ecdysone, 20E, 26-hydroxyecdysone and makisterone A $[1,6]$. Conjugation takes place at the hydroxy group on C-22 [6]; all the above ecdysteroids bear a hydroxy group at this position. A variety of other steroid molecules that do not contain this functional group at the correct position have been shown not to be conjugated by EGT [6]. As a result of this study, the range of substrates conjugated has now been extended to include 3DE. In keeping with the above criterion, 3DE also has a hydroxy group at C-22.

In all the previous studies mentioned above, no information was obtained on the relative ability of EGT to conjugate the different ecdysteroids. We have looked at the specificity of EGT for the molecules 3DE, ecdysone and 20E in this study because of their important positions in ecdysteroid metabolism. In Lepidoptera, ecdysteroids are produced and secreted by the pro- 
thoracic glands, in most species predominantly in the form of 3DE. This hormone is rapidly converted by the circulating enzyme 3 -dehydroecdysone $3 \beta$-reductase into ecdysone, the most abundant ecdysteroid form in the haemolymph. Finally, ecdysone is converted into $20 \mathrm{E}$ by ecdysone 20 -mono-oxygenase, an intracellular enzyme found in a range of different tissues. $20 \mathrm{E}$ is the most active ecdysteroid form in vivo, and is thought to be principally responsible for triggering the dramatic changes associated with ecdysis. Our kinetic data suggest that EGT targets the ecdysteroid precursors rather than the active from of the hormone; EGT has a 7-fold greater specificity for ecdysone than for $3 \mathrm{DE}$, but the enzyme conjugates $20 \mathrm{E}$ very poorly, more than 34 times less readily than $3 \mathrm{DE}$. This was somewhat surprising since it had been widely expected that $20 \mathrm{E}$ would be the optimal substrate, or at least that it would be used as efficiently as ecdysone. Mechanistically, it seems likely that $20 \mathrm{E}$ is a poor substrate because the hydroxy group at the C-20 position interferes in some way, possibly by steric hindrance, with the transfer of a sugar to the hydroxy group at C-22. Determination of the three-dimensional structure of the enzyme would be necessary to confirm this.

The ability of EGT to use a variety of UDP-sugars as sugar donors has also been evaluated previously. It was shown that both UDP-glucose and UDP-galactose are substrates of AcMNPV EGT in vitro [4]. However, again, no data were obtained on the relative specificity of EGT for either of these UDP-sugars. It was also observed that only ecdysteroid galactosides are formed in the haemolymph of AcMNPV-infected $S$. frugiperda insects. In contrast, Lymantria dispar MNPV (LdMNPV) EGT can use both UDP-glucose and UDP-galactose as substrates in vitro, and the conjugates constructed in the haemolymph of $L$. dispar insects infected with LdMNPV are exclusively glucosides [24]. The basis of this discrepancy is not known; it could either be that AcMNPV and LdMNPV EGTs exhibit a differential specificity for the two UDP-sugars, or that the difference is simply a reflection of the presence of different UDP-sugars in different insects. Our data show that there is no great difference in the ability of AcMNPV EGT to use either UDP-glucose or UDP-galactose. This indicates that only UDPgalactose is present in $S$. frugiperda haemolymph. We therefore suspect that only UDP-glucose is present in L. dispar haemolymph. It is not clear, however, why interspecies variation in UDP-sugar profiles should exist.

Kinetic analysis has also provided an insight into the mechanism of action of EGT. The Lineweaver-Burk plots shown in Figure 3 intersect in the second quadrant. This is indicative of an enzyme that acts via formation of a ternary complex [25], i.e. both the UDP-sugar and the ecdysteroid substrates are required to be bound to the enzyme at the same time in order for catalysis to occur. This is in agreement with the limited work that has been carried out on UDPGTs. As a result of substrate-inhibition studies, three specific kinetic mechanisms have been proposed for various UDPGT isoenzymes. These are a random-order ternary complex mechanism [12,26,27], a compulsory-order ternary complex mechanism [28] and a Theorell-Chance mechanism [28,29]. In the case of EGT, further work is necessary to allow a distinction to be made between the different forms of sequential mechanism.

Ternary complex formation is entirely in agreement with the hypothesis that UDPGTs have two major functional domains, a theory proposed as a result of structural and sequence homology studies made on these enzymes [30]. Each of these domains is thought to contain a binding site for one of the two substrates, enabling both substrate molecules to be bound to the enzyme at the same time. The domain at the $\mathrm{N}$-terminal half of the polypeptide chain is highly variable in sequence and believed to be responsible for aglycone binding. Evidence for this was provided by the results of domain-swap experiments with two enzyme forms that had different steroid specificities [30,31]. The domain located at the C-terminus of the protein is broadly conserved and thought to be involved in the binding of the common co-substrate UDP-glucuronic acid. Sequence alignment of UDPGTs with EGT enzymes has also led to the definition of such domains in these baculovirus proteins [5].

Most UDPGT enzymological studies have been performed on crude microsomal preparations because, owing to the extreme dependence of the UDPGTs on phospholipids, purified enzymes often have markedly different properties from the original membrane-bound proteins. Furthermore, UDPGTs are inherently difficult to purify because of their instability in the high detergent concentrations needed for solubilization. Both these problems are overcome with EGT because it is a secreted enzyme and therefore not dependent on the presence of phospholipids. Hence, it is relatively easy to obtain high-yield purified EGT preparations suitable for enzymological analysis. In this study we have shown that many of the physical and biochemical characteristics of EGT are similar to those of UDPGTs. Thus we propose that this baculovirus enzyme represents a valuable model system for studying the properties of this enzyme family.

We thank Professor H. H. Rees (University of Liverpool, U.K.) for the gift of 3dehydroecdysone. Thanks go to Dr. D. A. Fell (Oxford-Brookes University, U.K.), Dr. A. Cornish-Bowden (Centre National de la Recherche Scientifique, Marseille, France) and Professor J. M. Palmer (Imperial College, London, U.K.) for help with the enzyme kinetics, and to Professor H. H. Rees and Dr. D. P. Miller (Imperial College, London, U.K.) for helpful comments on the manuscript. This work was supported by the Biotechnology and Biological Sciences Research Council (U.K.) (grant no. PG028/0586)

\section{REFERENCES}

1 O'Reilly, D. R. and Miller, L. K. (1989) Science 245, 1110-1112

2 O'Reilly, D. R. and Miller, L. K. (1991) Bio/Technology 9, 1086-1089

3 Coudron, T. A., Law, J. H. and Koeppe, J. K. (1981) Trends Biochem. Sci. 6, 248-251

4 O'Reilly, D. R., Brown, M. R. and Miller, L. K. (1992) Insect Biochem. Mol. Biol. 22, 313-320

5 O'Reilly, D. R. (1995) Insect Biochem. Mol. Biol. 25, 541-550

6 O'Reilly, D. R., Howarth, O. W., Rees, H. H. and Miller, L. K. (1991) Insect Biochem. 21, 795-801

7 Warren, J. T., Steiner, B., Dorn, A., Pak, M. and Gilbert, L. I. (1986) J. Liq. Chromatogr. 9, 1759-1782

8 Thompson, M. J., Feldlaufer, M. F., Lozano, R., Rees, H. H., Lusby, W. R., Svoboda, J. A. and Wilzer, K. R. (1987) Arch. Insect Biochem. Physiol. 4, 1-15

9 Barker, G. C., Chitwood, D. J. and Rees, H. H. (1990) Invertebr. Reprod. Dev. 18, $1-11$

10 Burchell, B. and Coughtrie, M. (1989) Pharmacol. Ther. 43, 261-289

11 Erps, L. T., Ritter, J. K., Hersh, J. H., Blossom, D., Martin, N. C. and Owens, I. S. (1994) J. Clin. Invest. 93, 564-570

12 Vessey, D. A. and Zakim, D. (1972) J. Biol. Chem. 247, 3023-3028

13 Puig, J. F. and Tephly, T. R. (1986) Mol. Pharmacol. 30, 558-565

14 Vaughn, J. L., Goodwin, R. H., Tompkins, G. J. and McCawley, P. (1977) In Vitro 13, 213-217

15 O'Reilly, D. R., Miller, L. K. and Luckow, V. A. (1992) Baculovirus Expression Vectors; A Laboratory Manual, Oxford University Press, Oxford

16 Cornish-Bowden, A. (1995) Analysis of Enzyme Kinetic Data, Oxford University Press, Oxford

17 Laemmli, U. K. (1970) Nature (London) 227, 680-685

18 Sambrook, J., Fritsch, E. F. and Maniatis, T. (1989) Molecular Cloning: A Laboratory Manual, Cold Spring Harbor Laboratory Press, Cold Spring Harbor, NY

19 Buck, J. B. (1953) in Insect Physiology (Roeder, R. D., ed.), pp. 147-190, Chapman and Hall, London

20 O'Reilly, D. R. and Miller, L. K. (1990) J. Virol. 64, 1321-1328

21 Peters, W. H. M., Jansen, P. L. M. and Nauta, H. (1984) J. Biol. Chem. 259, $1701-1705$ 
22 Peters, W. H. M., Jansen, P. L. M., Cuypers, H. T. M., de Abreu, R. A. and Nauta, H. (1986) Biochim. Biophys. Acta 873, 252-259

23 Bruni, S. and Chang, T. M. S. (1995) Artif. Organs 19, 449-457

24 Kelly, T. J., Park, E. J., Masler, C. A. and Burand, J. P. (1992) Eur. J. Entomol. 92, $51-61$

25 Cornish-Bowden, A. (1995) Fundamentals of Enzyme Kinetics, Portland Press, London

26 Falany, C. N., Green, M. D. and Tephly, T. R. (1987) J. Biol. Chem. 262, 1218-1222

27 Yin, H. Q., Bennett, G. and Jones, J. P. (1994) Chem.-Biol. Interact. 90, 47-58

28 Koster, A. S. and Noordhoek, J. (1983) Biochim. Biophys. Acta 761, 76-85

29 Rao, M. L., Rao, G. S. and Breuer, H. (1976) Biochim. Biophys. Acta 452, 89-100

30 Mackenzie, P. I. (1990) J. Biol. Chem. 265, 3432-3435

31 Ritter, J. K., Sheen, Y. Y. and Owens, I. S. (1990) J. Biol. Chem. 265, 7900-7906

Received 9 October 1997/24 November 1997; accepted 4 December 1997 